\title{
Comunicação
}

[Communication]

\section{Mensurações ultrassonográficas da cisterna da glândula mamária de caprino transgênico}

\author{
[Ultrasound measurements of the mammary gland of transgenic hormone-induced \\ lactating goat] \\ C.H.S. Melo, F.C. Sousa, A.C.A. Teles Filho, R.R. Moura, E.S. Albuquerque, A.F. Pereira, \\ L.M. Melo, V.J.F. Freitas, D.I.A. Teixeira* \\ Faculdade de Veterinária - Universidade Estadual do Ceará - Fortaleza, CE
}

A ultrassonografia modo $\mathrm{B}$ em tempo real constitui uma técnica de diagnóstico por imagem cuja utilização, na prática veterinária, mostra-se em constante evolução. Destaca-se por permitir a avaliação e o estudo dos órgãos internos de forma constante, eficaz e segura, bem como a visualização completa e não invasiva das estruturas anatômicas (Simões, 2008). O estudo das estruturas internas da glândula mamária in vivo é realizado, principalmente, por meio de técnicas de ultrassonografia e mensurações indiretas do úbere. No entanto, a realização das medidas morfológicas por ferramentas tradicionais, compasso, fita métrica e paquímetro, são de acurácia limitada.

Como a saúde do úbere condiciona em grande parte a qualidade do leite, a ultrassonografia mamária pode ser utilizada como uma importante ferramenta que auxilia a avaliação dessa estrutura. Pode ser empregada no diagnóstico de diversas patologias (Flöck e Winter, 2006) e estimação da produção de leite (Nudda et al., 2000; Ayadi et al., 2003; Wójtowski et al., 2006). Os principais parâmetros ultrassonográficos utilizados para estimar a produção de leite em ruminantes são: comprimento maior, comprimento menor e área da cisterna (Nudda et al., 2000; Ayadi et al., 2003).

Em vista dos atuais trabalhos envolvendo caprinos transgênicos, denotando a fundamental importância da glândula mamária como principal biorreator na produção de proteínas exógenas
(Freitas et al., 2010), é essencial estudar todo o desenvolvimento da glândula mamária desses animais. Nesse sentido, o objetivo deste trabalho é avaliar, por meio da mensuração ultrassonográfica da glândula mamária, a produção de leite e o desenvolvimento morfológico do úbere do animal transgênico.

O experimento foi aprovado pelo Comitê de Ética de Uso de Animais da Universidade Estadual do Ceará (n. 12/2005) e pela Comissão Técnica Nacional de Biossegurança (CTNBio, $n$. 694/2006). Para tanto, foram utilizadas duas cabras da raça Canindé, não prenhes e não lactantes, com aproximadamente nove meses de idade, sendo um animal transgênico para hGCSF e um controle. Ambas as fêmeas foram mantidas em regime de manejo semi-intensivo.

Para a realização do experimento, os animais foram submetidos a um protocolo de indução da lactação. Para tanto, as cabras receberam sete aplicações, em dias alternados (D1, D3, D5, D7, D9, D11, D13), por via intramuscular, de $0,25 \mathrm{mg} / \mathrm{kgPV}$ de cipionato de estradiol e $0,75 \mathrm{mg} / \mathrm{kgPV}$ de progesterona. A partir do D14 foi administrado $0,40 \mathrm{mg} / \mathrm{kgPV}$ de predinisolona por via intramuscular, durante três dias consecutivos. Massagem externa da glândula mamária foi realizada diariamente, com início no D5, para estimular a lactogênese.

As avaliações ultrassonográficas foram executadas com o auxílio de um equipamento em tempo real, modo B, Falcon 100 (Pie Medical ${ }^{\circledR}$,

Recebido em 26 de agosto de 2011

Aceito em 23 de novembro de 2011

*Autor para correspondência (corresponding author)

E-mail: darcioitalo@yahoo.com.br 
Maastricht, Holanda), acoplado a um transdutor convexo de $5 \mathrm{MHz}$. Os exames foram realizados diariamente, do primeiro ao $17^{\circ}$ dia de lactação, e, semanalmente, do $18^{\circ}$ ao $80^{\circ}$ dia. O aparelho de ultrassom foi conectado a um computador portátil, e as imagens foram registradas com o auxílio de um programa computacional, destinado à gravação de vídeos. Nas imagens gravadas, os seguintes parâmetros: área, comprimento maior e menor da cisterna foram mensurados em um programa computacional (Image J, National Institutes of Health, Millersville, EUA), destinado à análise de imagens. Para realizar as mensurações, uma calibração prévia foi realizada; dessa forma, foi estabelecido que, para a frequência de $5 \mathrm{MHz}$, $1 \mathrm{~mm}$ equivalia a 16,732 pixels e $18 \mathrm{~cm}^{2}$.

Com relação à técnica ultrassonográfica utilizada, o animal foi contido em estação e a sonda posicionada caudalmente à inserção do teto, formando um ângulo de aproximadamente $30^{\circ}$, apontando para a cisterna do úbere. Para facilitar o contato e eliminar o ar entre a parede do úbere e o transdutor, este foi lubrificado com gel de carboximetilcelulose. Com o início da lactação e até a secagem, os animais foram submetidos à ordenha manual de forma higiênica. Para tanto, o volume de leite $(\mathrm{mL})$ presente em cada teta foi colhido em tubos graduados estéreis, permitindo a quantificação individual.

Os resultados foram analisados pelo programa Statística 7.0, e as médias expressas em média \pm erro-padrão. A relação entre a produção de leite e a área da cisterna mensurada por ultrassonografia foi calculada e descrita por uma equação de regressão linear, bem como pelo coeficiente de correlação. Os animais iniciaram a lactação no D16 do protocolo de indução hormonal, de acordo com o período esperado. A produção de leite total foi de 1,8 litros no animal transgênico e 9,0 litros no controle, estando dentro do valor esperado. As medidas obtidas por ultrassonografia podem ser observadas na Tab. 1 .

Nas 92 imagens avaliadas, o parênquima glandular (alta ecogenicidade) e o conteúdo da cisterna da glândula (anecoico) foram claramente visualizados em todos os exames. A cisterna do úbere caracterizou-se anecogênica e suas paredes eram irregulares em razão de aberturas dos grandes ductos lactíferos (Fig. 1).

As medidas do comprimento menor e comprimento maior da cisterna, respectivamente, apresentaram alta correlação positiva com $o$ volume de leite produzido, tanto para o animal transgênico $\left(\mathrm{Y}=-57,817+191,858^{*} \mathrm{x}, \mathrm{r}=0,94\right.$; $\mathrm{Y}=-24,816+38,220 * \mathrm{x}, \mathrm{r}=0,95)$ quanto para $\mathrm{o}$ controle $\left(\mathrm{Y}=-139,111+314,576^{*} \mathrm{x}, \mathrm{r}=0,97 ; \mathrm{Y}=\right.$ $\left.88,189+97,775^{*} \mathrm{x}, \mathrm{r}=0,98\right)$.

O comprimento maior e o comprimento menor máximo da cisterna foram, respectivamente, de $5,14 \mathrm{~cm}$ e $1,36 \mathrm{~cm}$ para o animal transgênico, e de $7,28 \mathrm{~cm}$ e $2,25 \mathrm{~cm}$ para o controle.

A relação entre a área média da cisterna e a produção de leite foi expressa em uma curva de regressão linear, e o coeficiente de correlação foi alto e positivo para o animal transgênico $(\mathrm{Y}=-1,1314+10,8538 * \mathrm{x} ; \mathrm{r}=0,97)$ e o controle $(\mathrm{Y}=-21,7551+18,3634 * x ; r=0,97)$.

As medidas do comprimento menor e comprimento maior da cisterna, respectivamente, apresentaram alta correlação positiva com o volume de leite produzido, tanto para o animal transgênico $\left(\mathrm{Y}=-57,817+191,858^{*} \mathrm{x}, \mathrm{r}=0,94\right.$; $\mathrm{Y}=-24,816+38,220 * \mathrm{x}, \mathrm{r}=0,95)$ quanto para $\mathrm{o}$ controle $(\mathrm{Y}=-139,111+314,576 * \mathrm{x}, \quad \mathrm{r}=0,97$; $\left.\mathrm{Y}=-88,189+97,775^{*} \mathrm{x}, \mathrm{r}=0,98\right)$.

Tabela 1. Médias (土erro-padrão) da produção total de leite e mensurações ultrassonográficas de caprinos da raça Canindé transgênico para o hG-CSF e controle em lactação induzida

\begin{tabular}{lcc}
\hline Variável & Transgênica & Controle \\
\hline Produção total $(\mathrm{L})$ & 1,8 & 9,0 \\
Área da cisterna $\left(\mathrm{cm}^{2}\right)$ & $5,23 \pm 1,27$ & $11,00 \pm 2,58$ \\
Comprimento maior da cisterna & $2,11 \pm 0,35$ & $3,55 \pm 0,49$ \\
Comprimento menor da cisterna & $0,59 \pm 0,07$ & $1,27 \pm 0,15$ \\
\hline
\end{tabular}




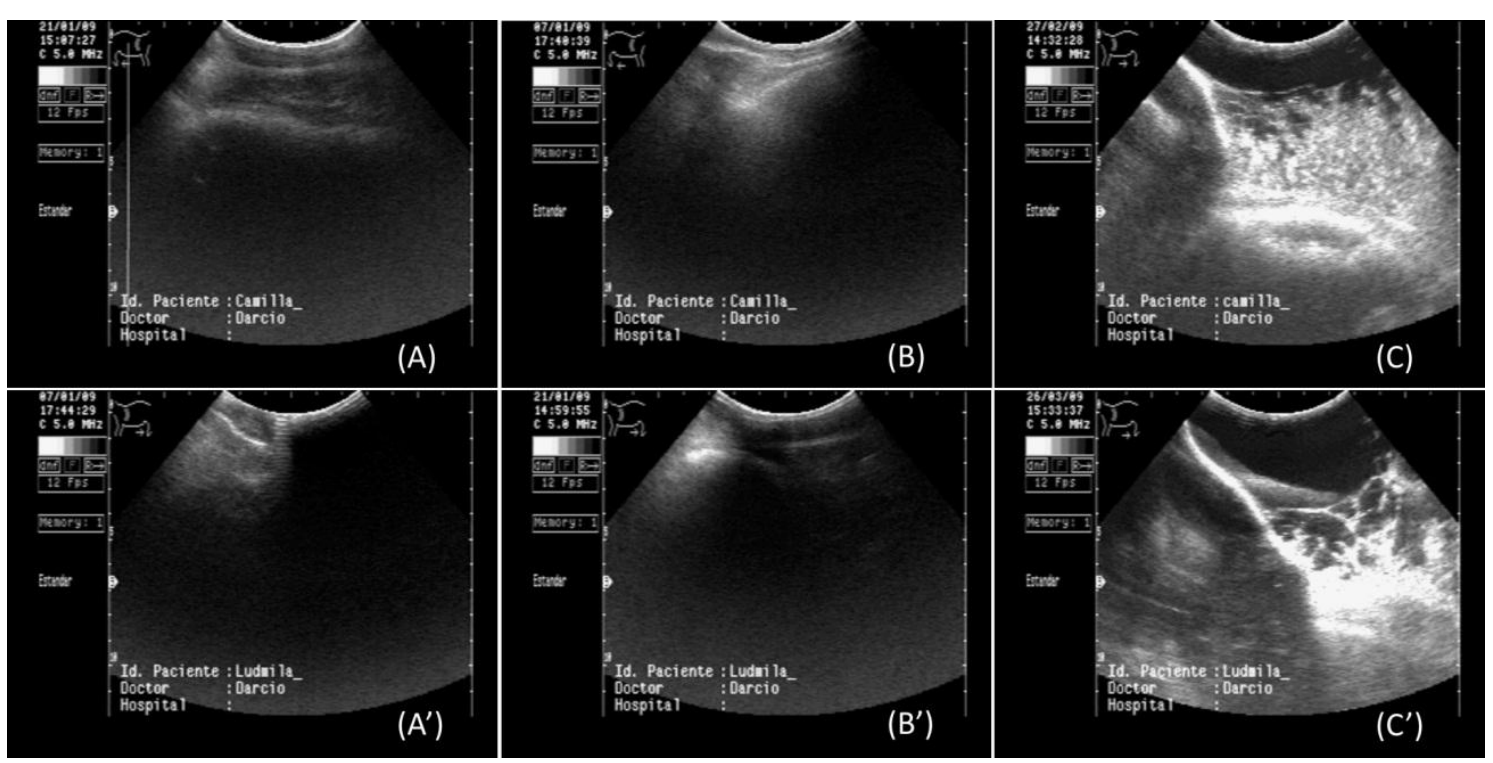

Figura 1. Cabra. Imagens ultrassonográficas da glândula mamária de animal transgênico (A, B e C) e controle (A', B' e C') submetidos a protocolo de indução da lactação no início do tratamento (A e A'), no primeiro dia da lactação (B e B') e no dia de maior produção de leite (C e C').

As medidas do comprimento menor e comprimento maior da cisterna, respectivamente, apresentaram alta correlação positiva com o volume de leite produzido, tanto para o animal transgênico $\left(\mathrm{Y}=-57,817+191,858^{*} \mathrm{x}, \mathrm{r}=0,94\right.$; $\mathrm{Y}=-24,816+38,220 * \mathrm{x}, \mathrm{r}=0,95)$ quanto para $\mathrm{o}$ controle $\left(\mathrm{Y}=-139,111+314,576^{*} \mathrm{x}, \mathrm{r}=0,97 ; \mathrm{Y}=\right.$ $\left.88,189+97,775^{*} \mathrm{x}, \mathrm{r}=0,98\right)$.

O comprimento maior e o comprimento menor máximo da cisterna foram, respectivamente, de $5,14 \mathrm{~cm}$ e $1,36 \mathrm{~cm}$ para o animal transgênico, e de $7,28 \mathrm{~cm}$ e $2,25 \mathrm{~cm}$ para o controle.

A relação entre a área média da cisterna e a produção de leite foi expressa em uma curva de regressão linear, e o coeficiente de correlação foi alto e positivo para $\mathrm{o}$ animal transgênico $\left(\mathrm{Y}=-1,1314+10,8538^{*} \mathrm{x} ; \mathrm{r}=0,97\right)$ e o controle $(\mathrm{Y}=-21,7551+18,3634 * x ; r=0,97)$.

Apesar da menor quantidade de leite produzida pelo animal transgênico, 1,8 litros, em relação ao controle, 9,0 litros, ambos produziram valores dentro do esperado. Todas as medidas ultrassonográficas foram possíveis de serem realizadas em ambos os animais. Tais medidas já foram verificadas no úbere de ovinos, bovinos e caprinos (Nudda et al., 2000; Ayadi et al., 2003; Salama et al., 2004).
Em relação às técnicas de ultrassonografia propostas por Wójtowski et al. (2006), a metodologia utilizada no presente estudo diferiu quanto ao posicionamento e à frequência do transdutor previamente estabelecida por tais autores. Apesar disso, os resultados obtidos foram satisfatórios e semelhantes aos observados em outros estudos (Nudda et al., 2000; Ayadi et al., 2003; Salama et al., 2004).

Neste estudo, observou-se que a mensuração da área, o comprimento maior e comprimento menor da cisterna apresentaram correlação significativa positiva com a produção de leite. Estes resultados assemelham-se aos encontrados na literatura (Salama et al., 2004; Wójtowski et al., 2006).

Em ovinos, Nudda et al. (2000), ao realizarem mensurações da área da cisterna do úbere, a partir de um transdutor setorial convexo de $3,5 \mathrm{MHz}$, verificaram um coeficiente de correlação com a produção leiteira de 0,81 . Ślósarz et al. (2002), ao mensurarem a cisterna da glândula mamária do úbere em ovinos com uma sonda convexa de $5 \mathrm{MHz}$, estimaram que a correlação com a produção de leite foi de 0,74 . 
Com relação às mensurações da cisterna realizadas por Salama et al. (2004), ao utilizarem uma sonda setorial de $5 \mathrm{MHz}$, obtiveram resultados semelhantes aos deste trabalho, em cabras Murciano-Granadina. Ainda, Wójtowski et al. (2002) relataram correlação semelhante em caprinos Polish White.
A técnica de ultrassonografia mostrou-se uma ferramenta simples e adequada para avaliar a cisterna do úbere de animais transgênicos.

Palavras-chave: caprino, úbere, ultrassonografia, transgênese

\begin{abstract}
Milk production of transgenic does was evaluated by ultrasound measurements of the mammary gland. Two Canindé goats, which were nine months of age were used in the trial, one non-transgenic or other transgenic for hG-CSF. For hormone-induced lactation, animals were given estradiol (0.25mg/kg, IM), progesterone $(0.75 \mathrm{mg} / \mathrm{kg}$, IM), and prednisolone $(0.4 \mathrm{mg} / \mathrm{kg}, \mathrm{IM})$. Ultrasonographic exams were carried out during milking, using a Falcon 100 ultrasound equipment with a $5 \mathrm{MHz}$ convex probe and were performed by the same operator. The results were expressed as mean \pm standard error. The maximum greater length and shorter length of the cistern were respectively $5.14 \mathrm{~cm}$ and $1.36 \mathrm{~cm}$ for the transgenic animal and $7.28 \mathrm{~cm}$ and $2.25 \mathrm{~cm}$ for non-transgenic, which is consistent with the maximum milk volume produced. The relationship between the average area of cisterns and milk yield was expressed as a linear correlation curve, with a correlation coefficient significantly positive for both transgenic ( $Y=-$ $1.1314+10.8538 * x ; \quad r=0.97)$ and non-transgenic $(Y=-21.7551+18.3634 * x ; \quad r=0.97)$ animals. In conclusion, the ultrasound is a practice and appropriate technique to evaluate the cisterns in ruminant udders in transgenic animal.
\end{abstract}

Keywords: goat, udder, ultrasonography, transgenesis

\section{REFERÊNCIAS}

AYADI, M.; CAJA, G.; SUCH, X.; KNIGHT, C.H. Use of ultrasonography to estimate cistern size and milk storage at different milking intervals in the udder of dairy cows. J. Dairy Res., v.70, p.1-7, 2003.

FLÖCK, M.; WINTER, P. Diagnostic ultrasonography in cattle with diseases of the mammary gland. Vet. J., v.171, p.314-321, 2006.

FREITAS, V.J.F.; TEIXEIRA, D.I.A.; MELO, L.M. et al. Generation of transgenic naturalized goats producing human granulocyte-colony stimulating factor (hG-CSF) in Brazil. Transgenic Res., v.19, p.146, 2010.

NUDDA, A.; PULINA, G.; VALLEBELLA, R. et al. Ultrasound technique for measuring mammary cistern size of dairy ewes. J. Dairy Res., v.67, p.101-106, 2000.

SALAMA, A.A.K.; CAJA, G.; SUCH, X. et al. Changes in cisternal udder compartment induced by milking interval in dairy goats milked once or twice daily. J. Dairy Sci., v.87, p.1181-1187, 2004.
SIMÕES, J. Os princípios físicos, a imagem e os artefatos na ecografia em modo B. 2008. Disponível em: 〈http://www.veterinaria.com.pt>. Acessado em: 11 jan. 2011.

ŚLÓSARZ, P.; WÓJTOWSKI, J.; GUT, A. et al. Preliminary results of application of ultrasound technique for estimation of milk yield in the sheep. Zesz. Nauk. Przegl. Hod., v.63, p.113-118, 2002.

WÓJTOWSKI, J.; JUNKUSZEW, A.; MILERSKI, M. et al. Application of ultrasound technique for cistern size measurement in dairy goats. Archiv. Tierzucht., v.4, p.382-388, 2006.

WÓJTOWSKI, J.; LÓSARZ, P.; GÓRECKI, M.; MALECHA, W. Ultrasound measurements of goat's mammary gland cisterns during lactation. Medycyna Wetrynaryjna, v.58, p.977-980, 2002. 